\title{
Effect of a charged boundary on electrophoresis: A sphere at an arbitrary position in a spherical cavity
}

\author{
Jyh-Ping Hsu *, Li-Hsien Yeh, Zheng-Syun Chen \\ Department of Chemical Engineering, National Taiwan University, Taipei 10617, Taiwan
}

Received 7 December 2006; accepted 11 January 2007

Available online 15 February 2007

\begin{abstract}
The effect of the presence of a charged boundary on the electrophoretic behavior of a particle is investigated by considering a sphere at an arbitrary position in a spherical cavity under conditions of low surface potential and weak applied electric field. Previous analyses are modified by using a more realistic electrostatic force formula and several interesting results, which are not reported in the literature, are observed. We show that the qualitative behavior of a particle depends largely on its position, its size relative to that of a cavity, and the thickness of the electric double layer. In general, the presence of a cavity has the effect of increasing the conventional hydrodynamic drag on a particle through a nonslip condition on the former. Also, a decrease in the thickness of the double layer surrounding a sphere has the effect of increasing the electrostatic force acting on its surface so that its mobility increases. However, this may not be the case when an uncharged particle in placed in a positively charged cavity, where the electroosmotic flow plays a role; for example, the mobility can exhibit a local maximum and the direction of electrophoresis can change.
\end{abstract}

(C) 2007 Elsevier Inc. All rights reserved.

Keywords: Electrophoresis; Effect of charged boundary; Sphere in spherical cavity

\section{Introduction}

The effect of the presence of a boundary on the electrophoretic behavior of a particle is of both fundamental and practical significance. The former is because solving the governing equations of electrophoresis is challenging even under drastically simplified conditions. The latter arises from electrophoresis often being conducted in a finite space, where the presence of a system boundary should not be neglected. Typical examples include capillary electrophoresis and electrophoresis of particles through a porous medium. The presence of a boundary can have a profound influence on the electrophoretic behavior of a particle because the concentration field, the electric field, and the flow field near the former, and consequently the forces acting on its surface, will be affected by the latter. In this case the classic electrophoresis theory of Smoluchowski [1] needs be modified to take the boundary effect into account, and

\footnotetext{
* Corresponding author. Fax: +886223623040.

E-mail address: jphsu@ntu.edu.tw (J.-P. Hsu).
}

many attempts have been made in the literature [2-8]. Among these, the idealized sphere-in-spherical-cavity model adopted by Zydney [2], where a sphere is located at the center of a spherical cavity, is of a simple and one-dimensional nature, yet the results obtained provide valuable insights into the boundary effect on electrophoresis. A more generalized model is to allow a sphere to be located at an arbitrary position in a spherical cavity [6]. In this case, the system under consideration becomes two-dimensional and the corresponding geometry is no longer totally symmetric. Under this condition, the formula used to calculate the electric force acting on a particle needs to be chosen carefully so that extraneous components are excluded $[9,10]$.

In addition to conventional hydrodynamic influence on a particle, the presence of a boundary can have other influences when it is charged. For instance, an electroosmotic flow field [2,11-14] and an osmotic pressure field [2,11-15] will be established near a charged surface. Also, a charge will be induced on an uncharged particle as it approaches a boundary. Apparently, these effects can play an important role in the determination of the mobility of a particle. Zydney [2] and Lee et al. [3,4], for 
instance, found that a charged cavity may alter the direction of the electrophoresis of a particle. Several other analyses of the influence of a charged boundary include, for example, a sphere moving along the axis of a cylindrical pore [11-13] and normal to a plane $[12,15]$ and a finite cylinder moving along the axis of a cylindrical pore [14].

In this study, the effect of the presence of a charged boundary on the electrophoretic behavior of a particle is investigated by considering a spherical particle at an arbitrary position in a spherical cavity under conditions of low surface potential and weak applied electric field. A new formula, which is more realistic than those used in the literature, is adopted to evaluate the electrostatic force acting on a particle. The influence of the key parameters, including the position of a particle, its relative size, and the thickness of the electric double layer, on the electrophoretic behavior of a particle is investigated.

\section{Theory}

Referring to Fig. 1, we consider the electrophoresis of a rigid, nonconductive, spherical particle of radius $a$ at an arbitrary position in a spherical, nonconductive cavity of radius $b$. The cylindrical coordinates $(r, \theta, z)$ are chosen with their origin placed at the center of the cavity; the center of the particle is at $z=m$. A uniform electric field $\mathbf{E}_{0}$ of strength $E_{0}$ in the $z$-direction is applied. Since the problem under consideration is $\theta$-symmetric, only the $(r, z)$ domain need be considered. We assume that $\mathbf{E}_{0}$ is relatively weak compared to the field established by a particle and/or by a boundary, and the surface potential is sufficiently low. The former is usually satisfied for conditions of practical significance and the latter is appropriate if the surface potential is lower than about $25 \mathrm{mV}$. Based on

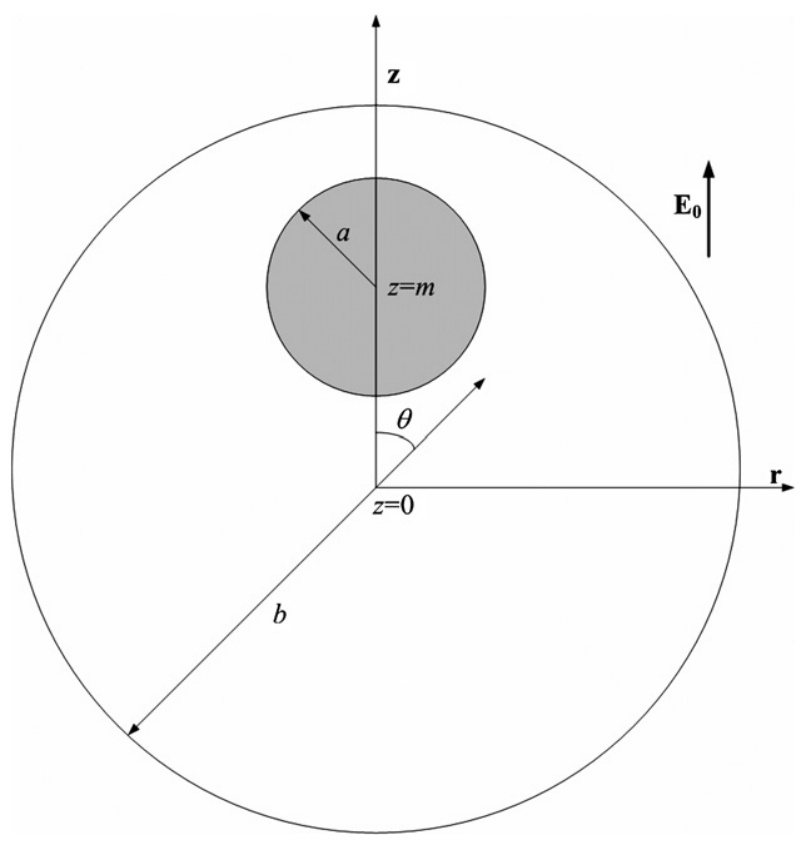

Fig. 1. The problem considered where a spherical particle of radius $a$ is placed at an arbitrary position in a spherical cavity of radius $b$. A uniform electric field $\mathbf{E}_{0}$ parallel to the $z$-direction is applied. The centers of the particle and the cavity are at $z=m$ and $z=0$, respectively, and $\theta$ is the solid angle. those assumptions, it can be shown that the electric potential of the present system, $\Psi$, can be described by [16]

$\nabla^{2} \Psi_{1}=\kappa^{2} \Psi_{1}$,

$\nabla^{2} \Psi_{2}=0$

where $\Psi=\Psi_{1}+\Psi_{2}, \Psi_{1}$ is the electrical potential in the absence of $\mathbf{E}_{0}$, and $\Psi_{2}$ is the electrical potential outside the particle arising from $\mathbf{E}_{0} . \nabla^{2}$ is the Laplace operator, $\kappa=$ $\left[\sum_{j} n_{j}^{0}\left(e z_{j}\right)^{2} / \varepsilon k_{\mathrm{B}} T\right]^{1 / 2}$ is the reciprocal Debye length, $n_{j}^{0}$ and $z_{j}$ being respectively the bulk number concentration and the valence of ionic species $j$, and $\varepsilon, e, k_{\mathrm{B}}$, and $T$ being respectively the permittivity of the liquid phase, the elementary charge, the Boltzmann constant, and the absolute temperature. The following boundary conditions are assumed,

$\Psi_{1}=\zeta_{\mathrm{a}}$ on particle surface,

$\Psi_{1}=\zeta_{\mathrm{b}}$ on cavity surface,

n. $\nabla \Psi_{2}=0$ on particle surface,

$\mathbf{n} \cdot \nabla \Psi_{2}=-E_{0} \cos \theta$ on cavity surface,

where $\mathbf{n}$ is the unit normal vector directed into the liquid phase. Equations (3) and (4) imply that the particle and the cavity are held at constant surface potentials $\zeta_{\mathrm{a}}$ and $\zeta_{\mathrm{b}}$, respectively. Equation (5) arises from the particle surface being nonconductive, and Eq. (6) implies that the local electric field on the cavity wall arises from the applied electric field [2].

If the liquid phase is an incompressible Newtonian fluid, then the flow field at steady state can be described by

$\nabla \cdot \mathbf{u}=0$,

$\eta \nabla^{2} \mathbf{u}-\nabla p=-\rho_{\mathrm{e}} \mathbf{E}_{0}$,

where $\mathbf{E}_{0}=-\nabla \Psi_{2}, \mathbf{u}, \eta$, and $p$ are respectively the velocity, the viscosity, and the pressure of the liquid phase, $-\rho_{\mathrm{e}} \mathbf{E}_{0}=\rho_{\mathrm{e}} \nabla \Psi_{2}$ is the electric body force acting on the fluid [9-12,17], and $\rho_{\mathrm{e}}=$ $\sum_{j} z_{j} e n_{j}^{0} \exp \left[-z_{j} e \Psi / k_{\mathrm{B}} T\right]$ is the space charge density. Let $U$ be the magnitude of the particle velocity in the $z$-direction and let $\mathbf{e}_{z}$ be the unit vector in the $z$-direction. Suppose that both the surface of a particle and that of the cavity are nonslip. Then the boundary conditions associated with Eqs. (7) and (8) are

$\mathbf{u}=U \mathbf{e}_{z} \quad$ on particle surface,

$\mathbf{u}=0 \quad$ on cavity surface.

In the present case only the $z$-components of the forces acting on a particle need be considered. These include the electrostatic force and the hydrodynamic force. Applying our recent result $[9,10]$ to the present case, the $z$-component of the former, $F_{\mathrm{E}}$, can be calculated by $[11,12,14,15,17]$

$F_{\mathrm{E}}=\iint_{S} \sigma_{\mathrm{p}} E_{z} d S$,

where $S$ denotes the particle surface, $\sigma_{\mathrm{p}}=-\varepsilon \mathbf{n} \cdot \nabla \Psi_{1}$ is the charge density on $S$, and $E_{z}=-\partial \Psi_{2} / \partial z$ is the strength of the local external electric field in the $z$-direction. The hydrodynamic force acting on a particle in the $z$-direction, $F_{\mathrm{D}}$, com- 
prises the viscous force and the pressure. The former can be evaluated by [18]

$F_{\mathrm{D}}=\iint_{S} \eta \frac{\partial(\mathbf{u} \cdot \mathbf{t})}{\partial n} t_{z} d S+\iint_{S}-p n_{z} d S$,

where $\mathbf{t}$ is the unit tangential vector on $S, n$ is the magnitude of $\mathbf{n}$, and $t_{z}$ and $n_{z}$ are respectively the $z$-component of $\mathbf{t}$ and that of $\mathbf{n}$. At steady state, we have

$F_{\mathrm{E}}+F_{\mathrm{D}}=0$.

This expression can be used to evaluate the electrophoretic mobility of a particle. Directly solving an electrophoresis problem involves the calculation of $U$ through a trial-and-error procedure based on Eq. (13). This tedious procedure can be avoided by partitioning the present problem into two subproblems [19]. In the first subproblem a sphere moves with speed $U$ in the absence of $\mathbf{E}_{0}$, and in the second subproblem, $\mathbf{E}_{0}$ is applied, but the sphere is kept fixed. In the former, a conventional hydrodynamic force $F_{\mathrm{D}, 1}=-U D$ acts on the sphere, where the drag coefficient $D$ is positive and depends upon its geometry and the boundary effect. In the latter, both an electrostatic force $F_{\mathrm{E}}$ and a hydrodynamic force $F_{\mathrm{D}, 2}$ act on the sphere. $F_{\mathrm{D}, 2}$ arises from the motion of the mobile ions in the electrical double layer when an external electric field is applied. In the present case $F_{\mathrm{D}, 2}$ can be either a drag force or a driving force. Note that both $F_{\mathrm{E}}$ and $F_{\mathrm{D}, 2}$ are functions of $\kappa a$, $\lambda(=a / b)$, and the relative position of a sphere in a cavity; $F_{\mathrm{D}, 1}$ (or $D$ ) is a function of $\lambda$ and the relative position of a sphere in a cavity, but is independent of $\kappa a$. Substituting the relations $F_{\mathrm{D}}=F_{\mathrm{D}, 1}+F_{\mathrm{D}, 2}$ and $F_{\mathrm{D}, 1}=-U D$ into Eq. (13), we obtain

$U=\frac{F_{\mathrm{E}}+F_{\mathrm{D}, 2}}{D}$.

Applying the procedure used previously [10,14], $U$ can be obtained. For convenience, the scaled electrophoretic mobility, $U^{*}=U / U_{\text {ref }}$, is used in subsequent discussions, where $U_{\text {ref }}=\varepsilon \zeta_{\text {ref }} E / \eta$ is the electrophoretic velocity of an isolated particle having a constant surface potential $\zeta_{\text {ref }}=k_{\mathrm{B}} T / e$. Two representative cases are considered: a positively charged sphere with a scaled constant surface potential $\zeta_{\mathrm{a}}^{*}=e \zeta_{\mathrm{a}} / k_{\mathrm{B}} T=1$ in an uncharged cavity, and an uncharged sphere in an positively charged cavity with a scaled constant surface potential $\zeta_{\mathrm{b}}^{*}=e \zeta_{\mathrm{b}} / k_{\mathrm{B}} T=1$. Note that the linear nature of the present problem implies that the result for the case when both the sphere and the cavity are charged can be obtained by a linear combination of the results obtained from these two cases. According to Eq. (14), if we let $F_{\mathrm{E}}^{*}=F_{\mathrm{E}} / 6 \pi \eta a U_{\text {ref }}, F_{\mathrm{D}, 2}^{*}=F_{\mathrm{D}, 2} / 6 \pi \eta a U_{\text {ref }}$, and $D^{*}=D / 6 \pi \eta a$, then

$U^{*}=\frac{F_{\mathrm{E}}^{*}+F_{\mathrm{D}, 2}^{*}}{D^{*}}$.

The numerator and the denominator on the right-hand side of this expression can be interpreted respectively as the net driving force per unit applied electrical field and the drag force per unit velocity of the sphere when $\mathbf{E}_{0}$ is absent.

Often, the total electric potential $\Psi$ is used in Eqs. (8) and (11) in the literature. For example, the electric body force in
Eq. (8) is expressed as $-\rho_{\mathrm{e}} \mathbf{E}_{0}=\rho_{\mathrm{e}} \nabla \Psi$ and Eq. (11) becomes $[3,6-8,20]$

$F_{\mathrm{E}}=\iint_{S} \sigma_{\mathrm{p}} E_{z} d S=\iint_{S} \varepsilon\left(\frac{\partial \Psi}{\partial n}\right)\left(\frac{\partial \Psi}{\partial z}\right) d S$.

It should be pointed out that if the geometry of a problem is totally symmetric, these formulas are applicable to various types of charged conditions $[9,10]$. If this is not the case, then Eqs. (8) and (11) should be used. This is because if the geometry of a problem is not of totally symmetric nature, an extraneous electric body force $\rho_{\mathrm{e}} \nabla \Psi_{1}$ and an extraneous electrostatic force arising from the equilibrium electric potential $\iint_{S} \varepsilon\left(\partial \Psi_{1} / \partial n\right)\left(\partial \Psi_{1} / \partial z\right) d S$ will be considered, thereby leading to incorrect results $[9,10]$.

\section{Results and discussion}

FlexPDE [21], a differential equation solver based on a finite-element method, is adopted for the resolution of the governing equations and the associated boundary conditions. The applicability of the numerical procedure adopted is justified by Fig. 2, where the result for the case of an uncharged sphere at the center of a charged spherical cavity is presented [2]. This figure reveals that the performance of the software used in this study is satisfactory for the ranges of $\lambda$ and $\kappa a$ considered.

\subsection{Particle positively charged, cavity uncharged}

Let us consider first the case when a positively charged spherical particle is in an uncharged spherical cavity. Fig. 3 shows the variations of the scaled drag force coefficient $D^{*}$ and the scaled electrophoretic mobility $U^{*}$ as functions of $P$ at various $\lambda$ when $a$ is fixed, that is, the radius of a sphere is fixed. The corresponding variations in the scaled electrostatic force $F_{\mathrm{E}}^{*}$, the scaled excess hydrodynamic force $F_{\mathrm{D}, 2}^{*}$, and the scaled net driving force $\left(F_{\mathrm{E}}^{*}+F_{\mathrm{D}, 2}^{*}\right)$ acting on a sphere are illustrated in Fig. 4. For comparison, the result of Hsu et al. [6], in which the formula used to evaluate $F_{\mathrm{E}}^{*}$ is different from that used in this study, is also presented in Fig. 3b. Here, $P=100 m /(b-a) \%$ is a position parameter, which measures the relative position of a sphere. Note that $P=0$ and $100 \%$ represent respectively the case when a sphere is at the center of a cavity and that when the sphere touches the cavity. According to Fig. 3a, for a fixed $\lambda, D^{*}$ increases with the increase in $P$, which is expected since the larger the value of $P$, the closer a sphere to a cavity, and the more important the influence of the latter on the movement of the former. This figure also reveals that if $P$ is fixed, $D^{*}$ increases with the increase in $\lambda$, that is, the more significant the boundary effect the greater the hydrodynamic drag on an uncharged particle, which is expected. Fig. $3 \mathrm{~b}$ indicates that, for a fixed $\lambda, U^{*}$ decreases monotonically with the increase in $P$, and $U^{*} \rightarrow 0$ as $P \rightarrow 100 \%$, that is, as the particle touches the cavity. This is expected because, as $P$ increases, the effect of the viscous retardation force due to the presence of the cavity becomes significant, and the rate of increase of $D^{*}$ is always 

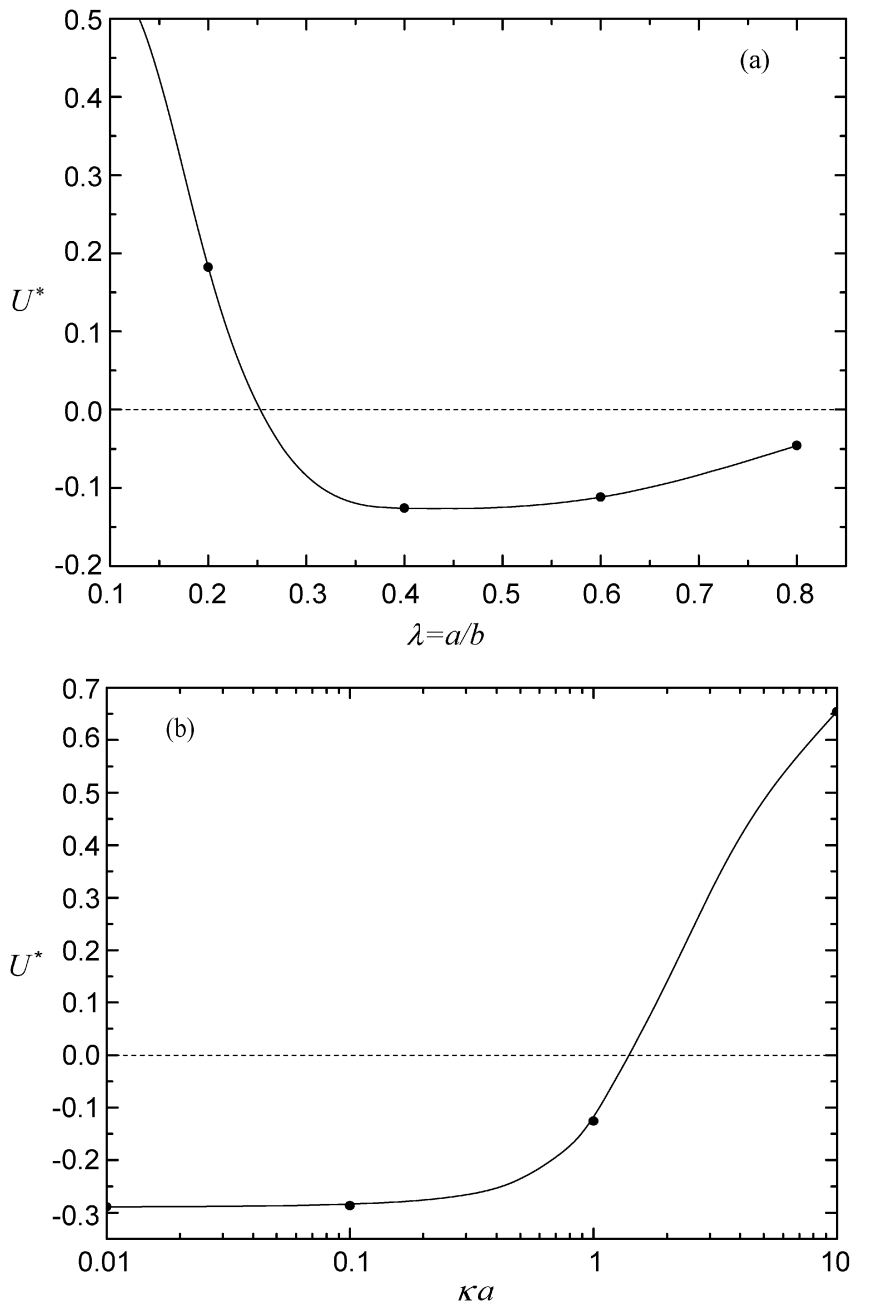

Fig. 2. Variation of scaled electrophoretic mobility $U^{*}$ (a) as a function of $\lambda(=a / b)$ at $\kappa a=1$ and (b) as a function of $\kappa a$ at $\lambda=0.4$ for the case where an uncharged sphere is placed at the center of a positively charged spherical cavity. Solid curve, present result; discrete symbols, result of Zydney [2]. Key: $\zeta_{\mathrm{a}}^{*}=0, \zeta_{\mathrm{b}}^{*}=1$.

higher than that of $\left(F_{\mathrm{E}}^{*}+F_{\mathrm{D}, 2}^{*}\right)$ shown in Fig. 4a. Fig. 3b reveals that the results of the present study at $P=0 \%$ and of that of Hsu et al. [6] are consistent. However, as $P$ becomes large, they are different both qualitatively and quantitatively, especially if $\lambda$ is large. In particular, if $\lambda$ is sufficiently large, the results of Hsu et al. [6] suggest that $U^{*}$ may increase with the increase of $P$. This is because for $P>0 \%$, an extra electric body force, $\rho_{\mathrm{e}} \nabla \Psi_{1}$, and an extra electrostatic force arising from the equilibrium electric potential, $\iint_{S} \varepsilon\left(\partial \Psi_{1} / \partial n\right)\left(\partial \Psi_{1} / \partial z\right) d S$, are included in the analysis $[9,10]$, and therefore, $U^{*}$ is overestimated. As can be seen in Fig. $4 \mathrm{a}, F_{\mathrm{E}}^{*}$ increases with the increase in $P$ and/or $\lambda$. The former is expected because when a charged particle approaches a neutral surface the contours of electrical potential (or double layer) surrounding the former will be distorted, and its surface charge density increases accordingly. The latter is a consequence of the combined effect of the squeeze of the applied electric field between the sphere and the cavity, and the increase in surface charge density as $\lambda$ increases [14]. Equation (12) can be rewritten in a scaled form as
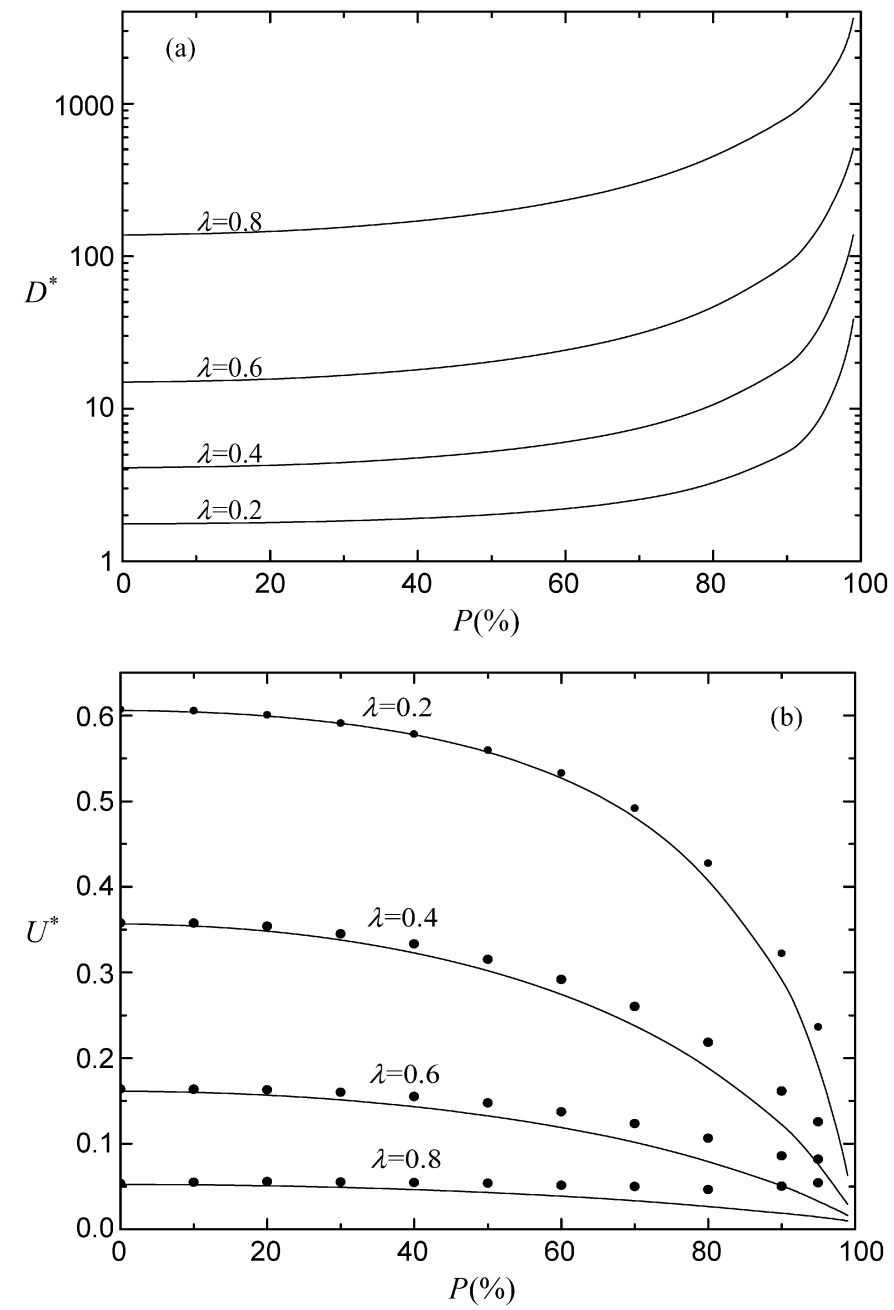

Fig. 3. Variation of (a) scaled hydrodynamic force coefficient $D^{*}$ and (b) scaled electrophoretic mobility $U^{*}$ as a function of $P$ at various values of $\lambda$ for the case of a positively charged sphere in an uncharged spherical cavity at $\zeta_{\mathrm{a}}^{*}=1$, $\zeta_{\mathrm{b}}^{*}=0$, and $\kappa a=1$. Solid curves, present result; discrete symbols, results of Hsu et al. [6].

$F_{\mathrm{D}, 2}^{*}=F_{\mathrm{D}, 2(v)}^{*}+F_{\mathrm{D}, 2(p)}^{*}$,

where $F_{\mathrm{D}, 2(v)}^{*}=\iint_{S} \eta[\partial(\mathbf{u} \cdot \mathbf{t}) / \partial n] t_{z} d S / 6 \pi \eta a U_{\text {ref }}$ and $F_{\mathrm{D}, 2(p)}^{*}$ $=\iint_{S}-p n_{z} d S / 6 \pi \eta a U_{\text {ref }}$ are respectively the scaled viscous term and the scaled pressure term of the scaled excess hydrodynamic force. According to Eq. (17), $F_{\mathrm{D}, 2}^{*}$ comprises a viscous term and a pressure term. Fig. $4 \mathrm{a}$ shows that $F_{\mathrm{D}, 2}^{*}$ is a retardation force when the boundary effect is unimportant, but it becomes a driving force when the boundary effect is significant. The latter arises from that the pressure term on the right-hand side of Eq. (17), $F_{\mathrm{D}, 2(p)}^{*}$, dominates [14]. Fig. $4 \mathrm{~b}$ indicates that $\left(F_{\mathrm{E}}^{*}+F_{\mathrm{D}, 2}^{*}\right)$ increases monotonically with an increase in $P$ and/or $\lambda$. This behavior of $\left(F_{\mathrm{E}}^{*}+F_{\mathrm{D}, 2}^{*}\right)$ is similar to that of $F_{\mathrm{E}}^{*}$ shown in Fig. $4 \mathrm{a}$, implying that $F_{\mathrm{E}}^{*}$ is the main driving force.

Fig. 5 shows the influence of $\kappa a$ and $P$ on the behavior of $U^{*}$. This figure reveals that, for a fixed $P, U^{*}$ increases monotonically with $\kappa a$, which can be explained by the qualitative behavior of $\left(F_{\mathrm{E}}^{*}+F_{\mathrm{D}, 2}^{*}\right)$ when $D^{*}=D^{*}(P, \lambda)$ is fixed. For the present case, both $F_{\mathrm{E}}^{*}$ and $F_{\mathrm{D}, 2}^{*}$ are strongly dependent upon the thickness of the double layer and the scaled net driving 

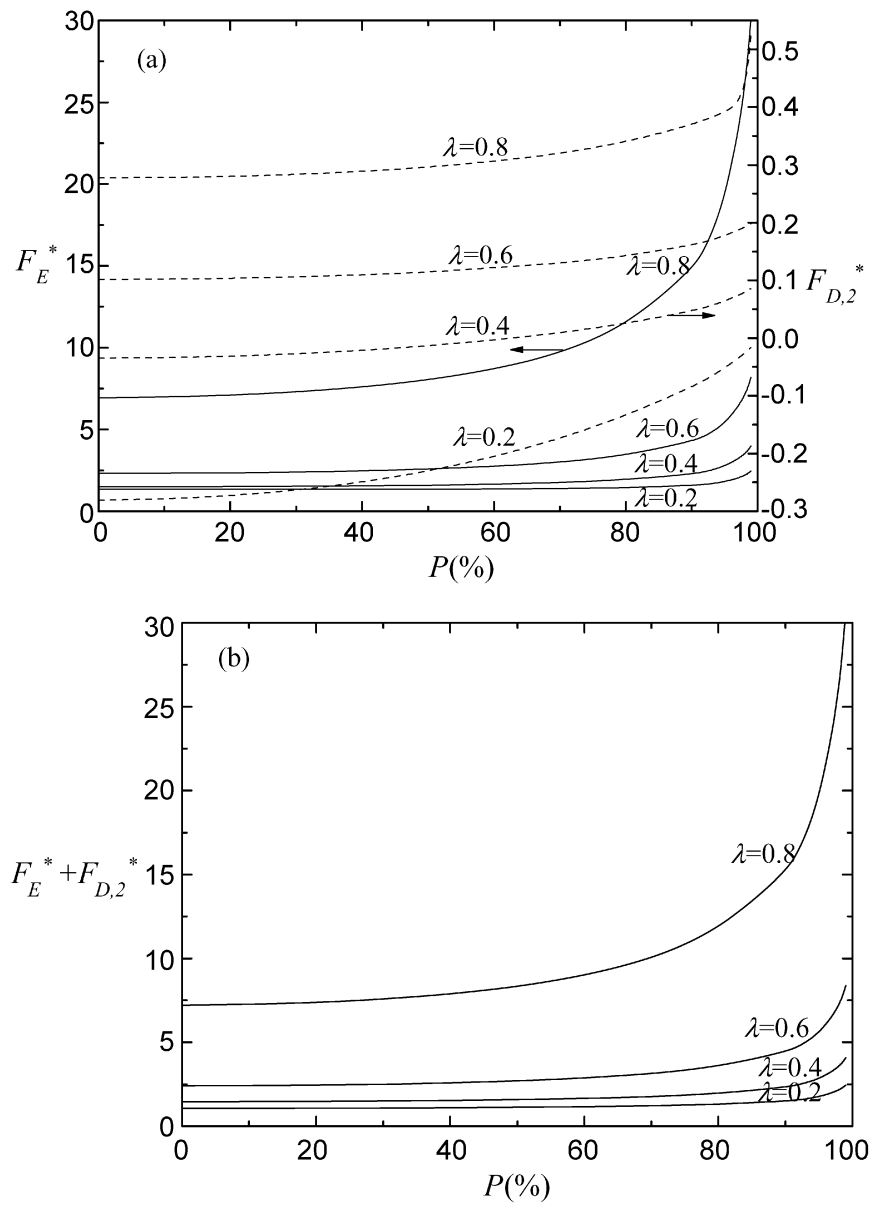

Fig. 4. Variation of (a) scaled electrostatic force $F_{\mathrm{E}}^{*}$ and scaled excess hydrodynamic force $F_{\mathrm{D}, 2}^{*}$ and (b) scaled net driving force $\left(F_{\mathrm{E}}^{*}+F_{\mathrm{D}, 2}^{*}\right)$ as functions of $P$ at various values of $\lambda$ for the case of Fig. 3 .

force $\left(F_{\mathrm{E}}^{*}+F_{\mathrm{D}, 2}^{*}\right)$, which is dominated by $F_{\mathrm{E}}^{*}$, increases with the increase in $\kappa a$. This is because the thinner the double layer surrounding a particle, the greater the absolute value of the gradient of the electrical potential on particle surface, the higher the surface charge density, and therefore, the greater the electrical driving force. Fig. $5 b$ suggests that if the double layer is thicker than the width of the gap between the sphere and the cavity when $P=0 \%, \kappa(b-a)<1$ or $\kappa a<\lambda(1-\lambda), U^{*}$ is insensitive to the variation in $\kappa a$. This is because if $\kappa(b-a)<1$, the double layer surrounding a particle is deformed by the cavity wall, and $F_{\mathrm{E}}^{*}$, which is much greater than $F_{\mathrm{D}, 2}^{*}$, depends weakly on $\kappa a$ [14]. Note that, for a fixed value of $\lambda$, the value of $\kappa a$ at which $U^{*}$ begins to increase rapidly increases with the increase in $P$. This is expected, since the larger the value of $P$, the closer a sphere to a cavity, and the more important the influence of the deformation of the double layer.

Fig. 6 shows the variations of $D^{*}$ and $U^{*}$ as a function of $\lambda$ at various values of $P$ when $\kappa a=1$. Fig. 6a suggests that $D^{*}$ increases monotonically with the increase in $\lambda$ and/or $P$, which is consistent with the results of Fig. 3a. Note that as $\lambda \rightarrow 0, D^{*} \rightarrow 1$, as is predicted be Stokes' law, which is based on a spherical particle in an unbounded fluid. As can be seen in Fig. 6b, for a fixed value of $P, U^{*}$ declines with the increase in $\lambda$, which is expected because the larger the value of $\lambda$
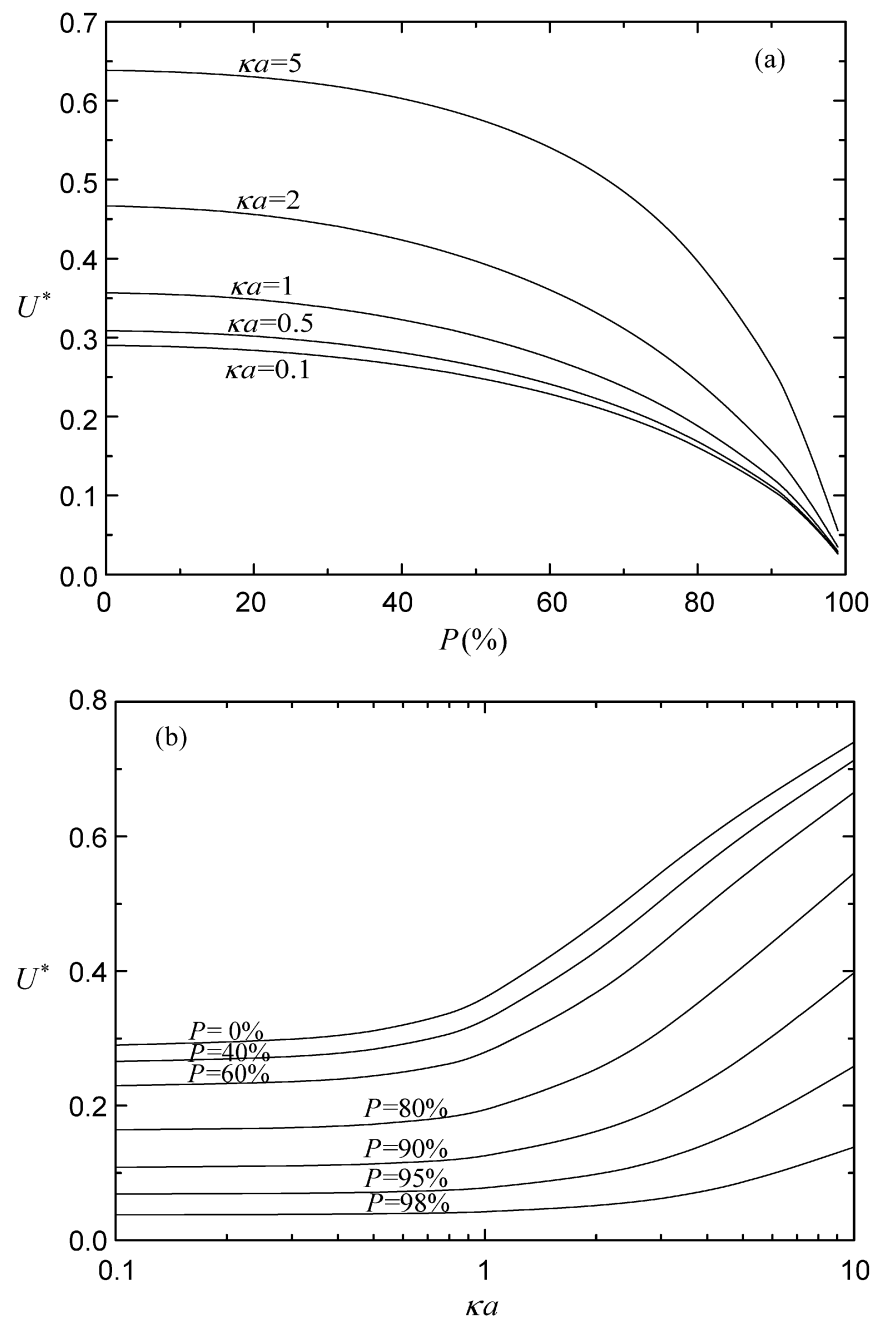

Fig. 5. Variation of (a) scaled electrophoretic mobility $U^{*}$ as a function of $P$ at various values of $\kappa a$ and (b) as a function of $\kappa a$ at various values of $P$. Key: $\zeta_{\mathrm{a}}^{*}=1, \zeta_{\mathrm{b}}^{*}=0$, and $\lambda=0.4$.

the more significant the presence of the boundary (cavity wall), which has the effect of retarding the movement of a particle.

\subsection{Particle uncharged, cavity positively charged}

If an uncharged sphere is placed in a positively charged cavity, an electroosmotic flow is generated due to the presence of the latter [2], and the former experiences an excess hydrodynamic force. The competition of this force with the electrostatic force makes the electrophoretic behavior of a sphere more complicated than for the case when a positively charged sphere is placed in an uncharged cavity. Also, a negative charge is induced on the surface of the sphere, and the corresponding electrostatic force acting on the particle is in the $-z$-direction. Fig. 7 shows the influence of $P$ and $\lambda$ on the behavior of $U^{*}$. It is interesting to note that the qualitative behavior of $U^{*}$ depends upon the level of $\lambda$ (or $\kappa a$ ). For example, if $\lambda$ is sufficiently large, $\left|U^{*}\right|$ declines wit the increase in $P$. If $\lambda$ takes a medium value ( $=0.4),\left|U^{*}\right|$ has a local maximum as $P$ varies. If $\lambda$ is sufficiently small, $U^{*}$ may change its sign from positive to negative as $P$ increases, and $\left|U^{*}\right|$ may have a local maximum if $P$ 

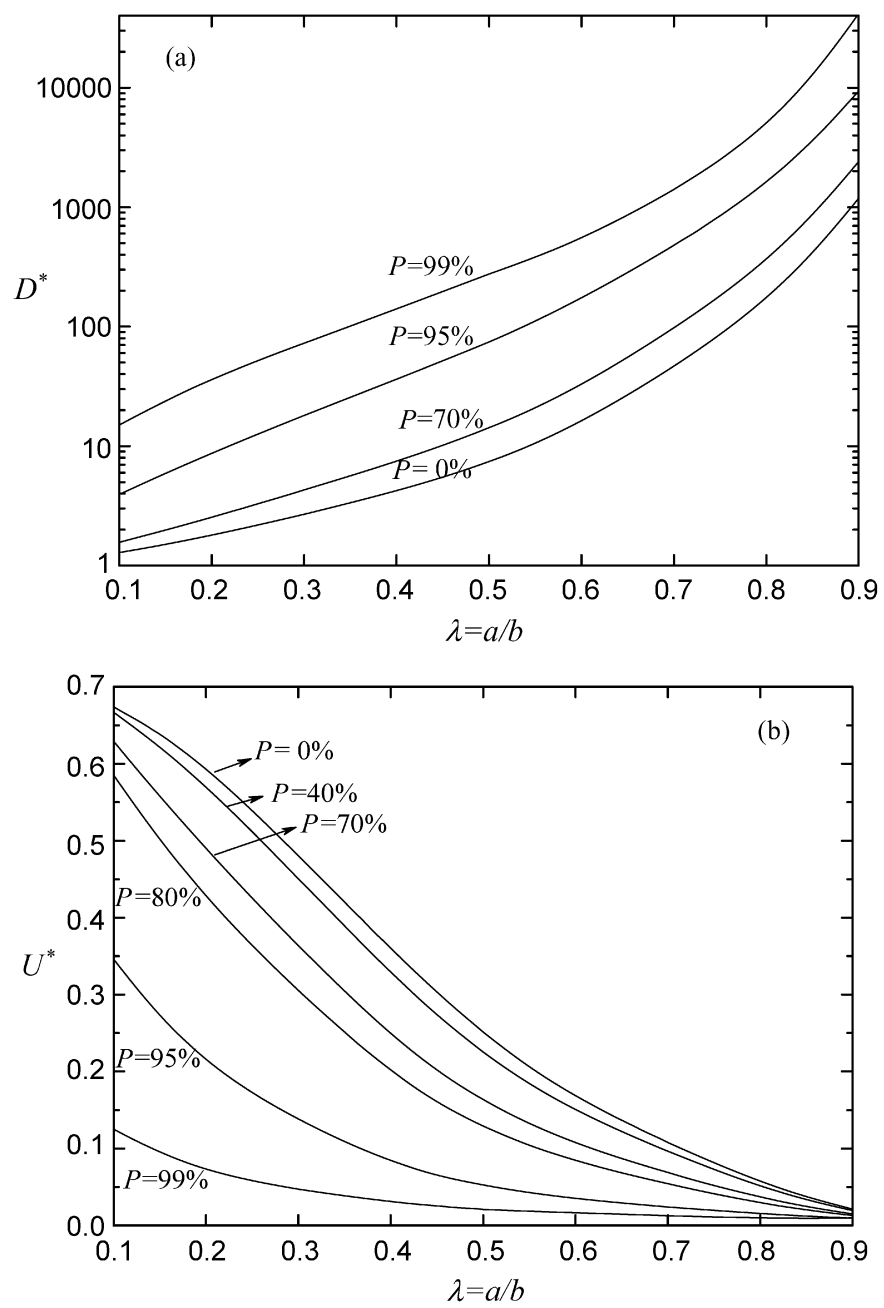

Fig. 6. Variation of (a) scaled hydrodynamic force coefficient $D^{*}$ and (b) scaled electrophoretic mobility $U^{*}$ as functions of $\lambda$ at various values of $P$. Key: $\zeta_{\mathrm{a}}^{*}=1, \zeta_{\mathrm{b}}^{*}=0$, and $\kappa a=1$.

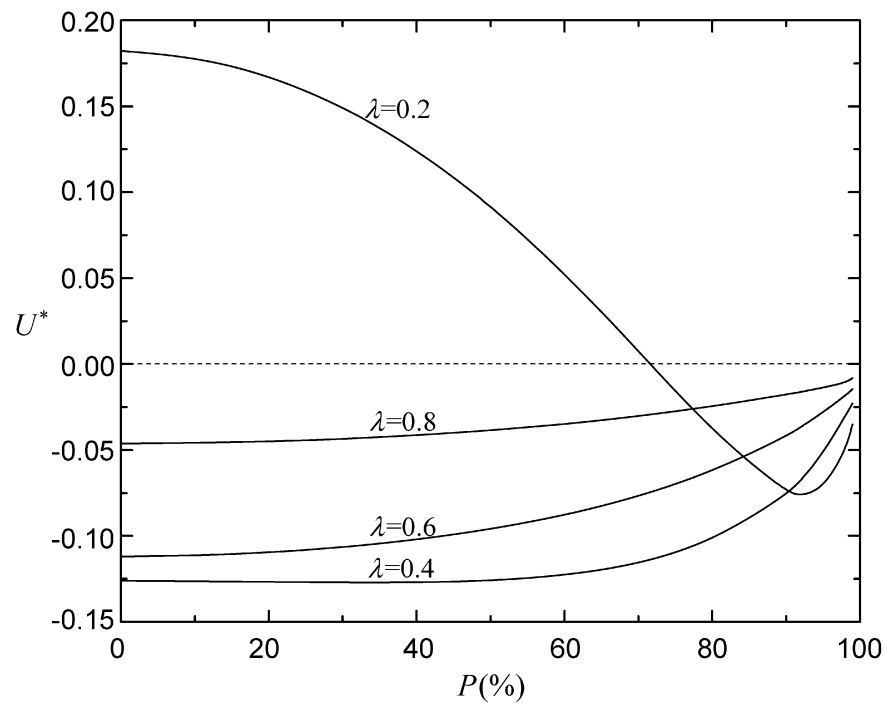

Fig. 7. Variation of scaled electrophoretic mobility $U^{*}$ as a function of $P$ at various values of $\lambda$. Key: $\zeta_{\mathrm{a}}^{*}=0, \zeta_{\mathrm{b}}^{*}=1$, and $\kappa a=1$.
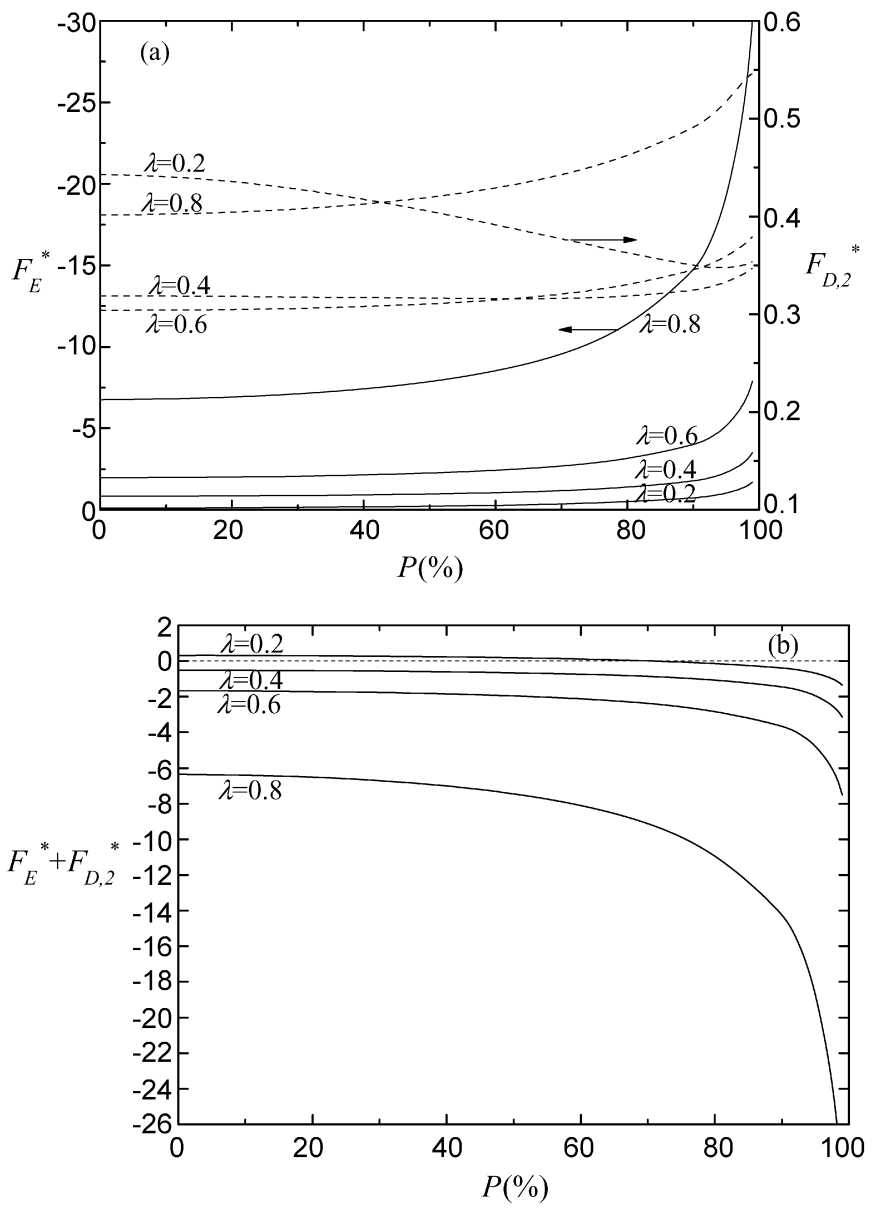

Fig. 8. Variation of (a) scaled electrostatic force $F_{\mathrm{E}}^{*}$ and scaled excess hydrodynamic force $F_{\mathrm{D}, 2}^{*}$ and (b) scaled net driving force $\left(F_{\mathrm{E}}^{*}+F_{\mathrm{D}, 2}^{*}\right)$ as a function of $P$ at various values of $\lambda$ for the case of Fig. 7 .

is sufficiently large ( $>72 \%$ ). Furthermore, $U^{*}$ is always negative except when $\lambda$ is sufficiently small $(<0.4)$. As is justified by Fig. 8, the occurrence of these phenomena is the net result of two competing driving forces, $F_{\mathrm{E}}^{*}$ and $F_{\mathrm{D}, 2}^{*}$, as $P$ and $\lambda$ vary, and the sign of $U^{*}$ depends upon which of the two forces dominates. If $\lambda$ is sufficiently large, the induced negative scaled electrostatic force $-F_{\mathrm{E}}^{*}$ dominates and the effect of the viscous retardation force due to the presence of the cavity becomes significant; that is, the rate of increase of $D^{*}$ as $P$ varies is higher than that of $\left(F_{\mathrm{E}}^{*}+F_{\mathrm{D}, 2}^{*}\right)$. This is similar to what is observed for the case when a positively charged sphere is in an uncharged cavity, except that $U^{*}$ is negative for the ranges of the parameters considered. For a medium value of $\lambda$ $(=0.4),-F_{\mathrm{E}}^{*}$ also dominates and the rate of increase of $D^{*}$ is smaller than that of $\left(F_{\mathrm{E}}^{*}+F_{\mathrm{D}, 2}^{*}\right)$ when $P$ is small, but the reverse is true when $P$ is large. If the boundary effect is relatively unimportant $(\lambda=0.2)$ and $P$ is small, the positive scaled excess hydrodynamic force $F_{\mathrm{D}, 2}^{*}$ becomes the dominant force and the increase in $D^{*}$ as $P$ increases leads to an decrease in $U^{*}$. On the other hand, if $P$ is sufficiently large ( $>72 \%),-F_{\mathrm{E}}^{*}$ increases rapidly with the increase in $P$ and can exceed $F_{\mathrm{D}, 2}^{*}$, and $U^{*}$ becomes negative. The occurrence of the local maximum in $\left|U^{*}\right|$ as $P$ increases from $72 \%$ arises from the rate of increase of $D^{*}$ always being slower than that of the scaled net driving 
force $\left(F_{\mathrm{E}}^{*}+F_{\mathrm{D}, 2}^{*}\right)$. However, if $P$ is sufficiently large, $D^{*}$ increases rapidly with the increase in $P$ and can further retard the motion of a sphere. Even if the boundary effect is relatively unimportant ( $\lambda$ is small), some unique electrophoretic behaviors are observed that have not been reported at other types of charged boundaries [11-15]. Note that if $P$ is sufficiently large, that is, a sphere is sufficiently close to a cavity, $\left|U^{*}\right|$ is close to zero, which is similar to the result shown in Fig. $3 \mathrm{~b}$ where a positively charged sphere is in an uncharged cavity, except that the sphere moves in the opposite direction. This behavior is reasonable because a nonslip boundary condition is assumed and the mobility of a particle should vanish when $P=100 \%$ regardless of the value of $\lambda$. All of the above observations are directly related to the results of $F_{\mathrm{E}}^{*}, F_{\mathrm{D}, 2}^{*}$, and $\left(F_{\mathrm{E}}^{*}+F_{\mathrm{D}, 2}^{*}\right)$ presented in Fig. 8. As can be seen in Fig. 8a, $\left|F_{\mathrm{E}}^{*}\right|$ increases with the increase in $P$ and/or $\lambda$. This can be explained by the same reasoning as that employed in the discussion of Fig. 4a. Note that $F_{\mathrm{E}}^{*}$ in Fig. 8a is negative; this is because a negative charge is induced on the sphere surface due to the presence of the positively charged pore. However, if both $\lambda$ and $P$ are sufficiently small, the amount of the induced charge decreases rapidly, and it vanishes as $\lambda \rightarrow 0$ and $P \rightarrow 0$. On the other hand, under the conditions assumed, $F_{\mathrm{D}, 2}^{*}$ is always positive and its qualitative behavior as $P$ varies depends upon the value of $\lambda$, as is illustrated in Fig. 8a. The former can be explained by that since the net volumetric flow rate of the fluid in the gap between a sphere and a cavity must vanish, an electroosmotic flow is present in that gap. For the present case, a clockwise (counterclockwise) vortex is generated on the right (left)-hand side of a sphere, and a negative pressure gradient in the $z$-direction (i.e., $d p / d z<0)$ is necessary to derive this flow. Therefore, referring to Eq. (17), both the viscous term $F_{\mathrm{D}, 2(v)}^{*}$ and the pressure term $F_{\mathrm{D}, 2(p)}^{*}$ involved in $F_{\mathrm{D}, 2}^{*}$ are positive, and so is $F_{\mathrm{D}, 2}^{*}$. This is different from the case when a positively charged sphere is in an uncharged cavity, where $F_{\mathrm{D}, 2}^{*}$ changes from negative to positive if the boundary effect is significant. The qualitative behavior of $F_{\mathrm{D}, 2}^{*}$ as $P$ varies depends upon the value of $\lambda$ as a consequence of the competition between $F_{\mathrm{D}, 2(v)}^{*}$ and $F_{\mathrm{D}, 2(p)}^{*}$. For example, if $\lambda$ is large (boundary effect is important), $F_{\mathrm{D}, 2}^{*}$ is dominated by $F_{\mathrm{D}, 2(p)}^{*}$ and increases with the increase in $P$. The former is because the electroosmotic flow is hindered by the cavity wall, and so is $F_{\mathrm{D}, 2(v)}^{*}$. The latter is because if $P$ is large, both the sphere and the fluid are subjected to a large increase in the negative pressure gradient mentioned previously. It is interesting to note that if the boundary effect is relatively unimportant $(\lambda=0.2), F_{\mathrm{D}, 2}^{*}$ declines with the increase in $P$ when $\kappa a=1$, but the reverse is true if $P$ is sufficiently large. This is because as $P$ increases, $F_{\mathrm{D}, 2}^{*}$ is mainly dominated by the decrease of $F_{\mathrm{D}, 2(v)}^{*}$, since the larger the value of $P$ the closer a sphere to a cavity, and the more important the influence of the latter on the movement of the former. However, the reverse is true due to a rapid increase in $F_{\mathrm{D}, 2(p)}^{*}$ when $P$ is sufficiently large. Fig. $8 \mathrm{~b}$ shows that if the boundary effect is significant ( $\lambda$ and/or $P$ is large), $\left(F_{\mathrm{E}}^{*}+F_{\mathrm{D}, 2}^{*}\right)$ is negative and its magnitude increases monotonically with an increase in $P$. On the other hand, if the boundary effect is unimportant, $\left(F_{\mathrm{E}}^{*}+F_{\mathrm{D}, 2}^{*}\right)$ is positive and decreases with the increase in $P$. These observations are different from those for the case when $\zeta_{\mathrm{a}}^{*}=1$ and $\zeta_{\mathrm{b}}^{*}=0$ shown in Fig. 4b, where the increase of $\left(F_{\mathrm{E}}^{*}+F_{\mathrm{D}, 2}^{*}\right)$ with an increase in $P$ arises from the increase of $F_{\mathrm{E}}^{*}$, implying that $F_{\mathrm{D}, 2}^{*}$ is the dominating force.

Some typical flow fields for the case of Fig. 7 are illustrated in Fig. 9. In Fig. 9a, where $P=70 \%$ and $\lambda=0.2$, a clockwise (counterclockwise) vortex is generated on the right (left)-hand side of a sphere. However, if $P$ is further increased to $95 \%$, in addition to a clockwise vortex, a counterclockwise vortex is also generated near the particle in the north pole of the cavity, as shown in Fig. 9b. The former arises because if the boundary effect is relatively unimportant and $P$ is not too large, the recirculation electroosmotic flow, which is generated due to the presence of the charged cavity, dominates the movement of a particle and leads to a positive mobility. The latter is because if $P$ is sufficiently large, the double layer near the cavity is distorted by the sphere and a negative charge is induced on the sphere surface, which leads to a negative scaled electric force $-F_{\mathrm{E}}^{*}$ acting on the sphere. Since this force dominates, the mobility of the sphere becomes negative. Figs. 9c and 9d reveal that if the boundary effect is important $(\lambda=0.7)$, a counterclockwise (clockwise) vortex is generated on the right (left)-hand side of a sphere. Again, this arises from the negative charge induced on the sphere surface, which leads to a negative mobility.

Fig. 10 shows the variations of $U^{*}, F_{\mathrm{E}}^{*}$, and $F_{\mathrm{D}, 2}^{*}$ as a function of $P$ at various values of $\kappa a$ for the case of a medium value of $\lambda$. As in the case of $\lambda=0.4$, Fig. 10a also indicates that the qualitative behavior of $U^{*}$ depends upon the level of $\kappa a$. For example, if $\kappa a$ is sufficiently small $(=0.1$ or 0.5$),\left|U^{*}\right|$ decreases with the increase in $P$. For a medium value of $\kappa a$ (=1), $\left|U^{*}\right|$ has a local maximum as $P$ varies, and if $\kappa a$ is large $(=2), U^{*}$ may change its sign from positive to negative as $P$ increases and $\left|U^{*}\right|$ may have a local maximum when $P$ is sufficiently large $(>83 \%)$. If $\kappa a$ is sufficiently large $(=5), U^{*}$ is always positive and declines with the increase in $P$. Also, $U^{*}$ is always negative, except when $\kappa a$ is sufficiently large. As in the case of $\lambda=0.4$, these behaviors arise from the competition between $F_{\mathrm{E}}^{*}$ and $F_{\mathrm{D}, 2}^{*}$, and the sign of $U^{*}$ depends upon which of these two forces dominates, as is justified by Fig. 10b. Note that the qualitative behavior of $U^{*}$ is similar to that observed in Fig. 7a, except when $\kappa a$ is sufficiently large. For example, if $\kappa a=5, U^{*}$ is always positive and decreases monotonically with the increase in $P$. This is because if the double layer is sufficiently thin, the magnitude of the negative induced electrostatic force is small and always smaller than that of the positive scaled excess hydrodynamic force; that is, $\left|F_{\mathrm{E}}^{*}\right|<F_{\mathrm{D}, 2}^{*}$, implying that $\left(F_{\mathrm{E}}^{*}+F_{\mathrm{D}, 2}^{*}\right)$ is dominated by $F_{D, 2}^{*}$ and the rate of increase of $D^{*}$ is faster than that of $\left(F_{\mathrm{E}}^{*}+F_{\mathrm{D}, 2}^{*}\right)$. Fig. $10 \mathrm{~b}$ reveals that, for a smaller $\kappa a$, the approach of a neutral sphere yields a more serious distortion of the double layer near a cavity, leading to a greater electrostatic force acting on the former. Fig. 10b also indicates that the larger the $\kappa a$ the greater the $F_{\mathrm{D}, 2}^{*}$, and if $\kappa a$ is sufficiently large, $F_{\mathrm{D}, 2}^{*}$ increases with the increase in $P$. The former is because if the double layer is thin, the electroosmotic recirculation flow surrounding a sphere is appreciable, and so are the drag acting on its surface and $F_{\mathrm{D}, 2(v)}^{*}$. The later is because that charge is induced on the surface of a 
(a)
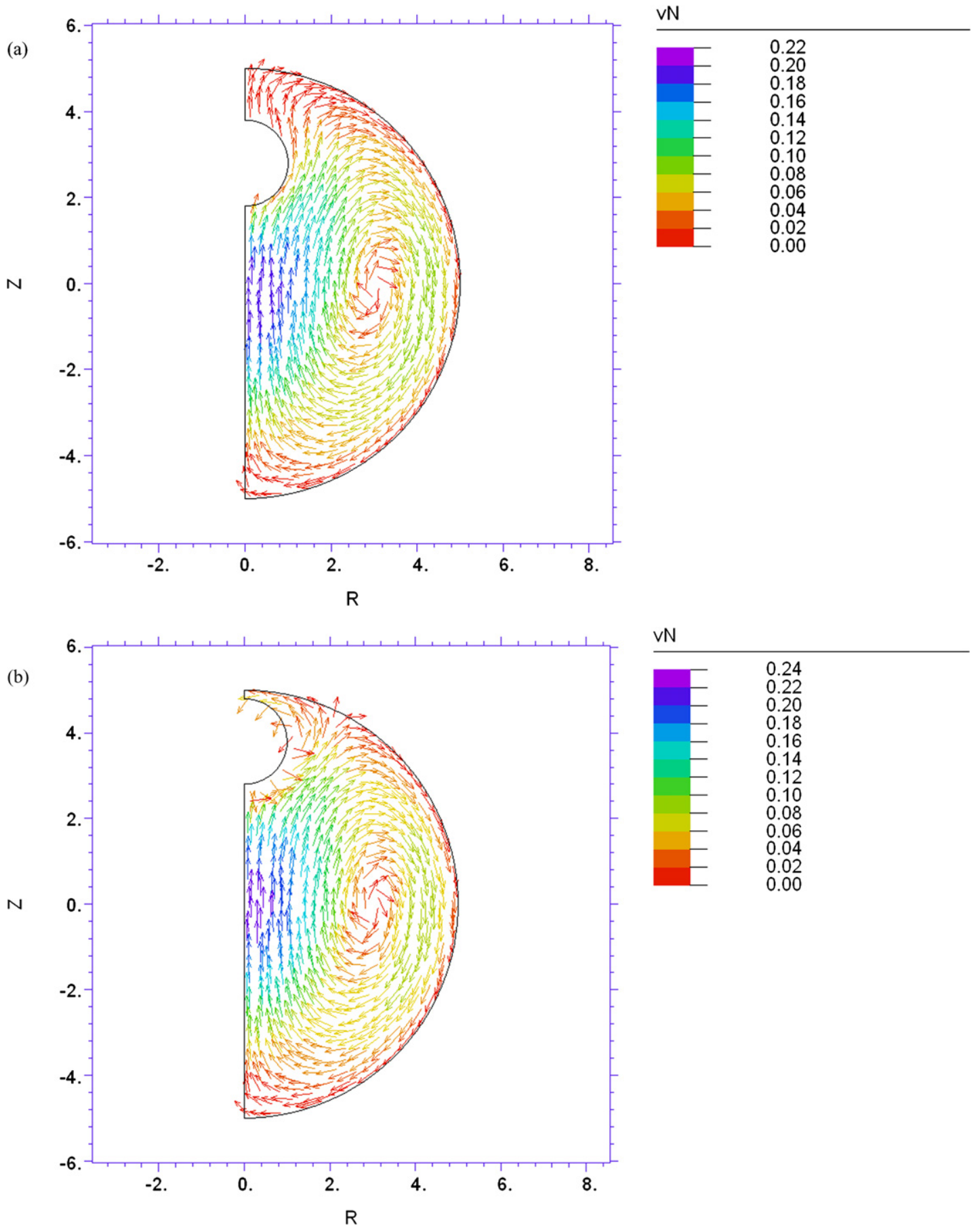

Fig. 9. Some typical flow fields for the case of Fig. 7. (a) $P=70 \%$ and $\lambda=0.2$; (b) $P=95 \%$ and $\lambda=0.2$; (c) $P=70 \%$ and $\lambda=0.7$; (d) $P=95 \%$ and $\lambda=0.7$.

sphere, and a pressure field is established, leading to a sudden rise in $F_{\mathrm{D}, 2(p)}^{*}$. On the other hand, if $\kappa a$ is sufficiently small, $F_{\mathrm{D}, 2}^{*}$ becomes small and insensitive to the variation in $P$. This is because if the electric double layer of a cavity is sufficiently thick, about the same amount of negative charge is induced on the surface of a sphere, regardless of its position. Consequently, $F_{\mathrm{D}, 2}^{*}$ is insensitive to the variation in $P$, since it is dominated by $F_{\mathrm{D}, 2(v)}^{*}$, which is mainly related to the electroosmotic flow. If a sphere is very close to the cavity surface, $F_{\mathrm{D}, 2(v)}^{*}$ declines due to a relatively large amount of negative charge being induced on its surface and a decrease in the electroosmotic flow. However, since $F_{\mathrm{D}, 2(p)}^{*}$ increases at the same time, $F_{\mathrm{D}, 2}^{*}$ remains roughly constant as $P$ varies.

The variation of $U^{*}, F_{\mathrm{E}}^{*}$, and $F_{\mathrm{D}, 2}^{*}$ as a function of $\kappa a$ at various values of $P$ when $\lambda=0.4$ is illustrated in Fig. 11. According to Fig. 11a, $U^{*}$ may change its sign from negative to positive. This arises from the competition between $F_{\mathrm{E}}^{*}$ and $F_{\mathrm{D}, 2}^{*} ;\left|F_{\mathrm{E}}^{*}\right|$ decreases but $F_{\mathrm{D}, 2}^{*}$ increases with the increase in $\kappa a$, as is shown in Fig. 11b. The value of $\kappa a$ at which $U^{*}$ changes its sign increases with the increase in $P$. This is because as $P$ increases, $F_{\mathrm{D}, 2}^{*}$ increases rapidly due to the presence of the cavity; that is, the rate of increase of $F_{\mathrm{D}, 2(p)}^{*}$ is always much higher 

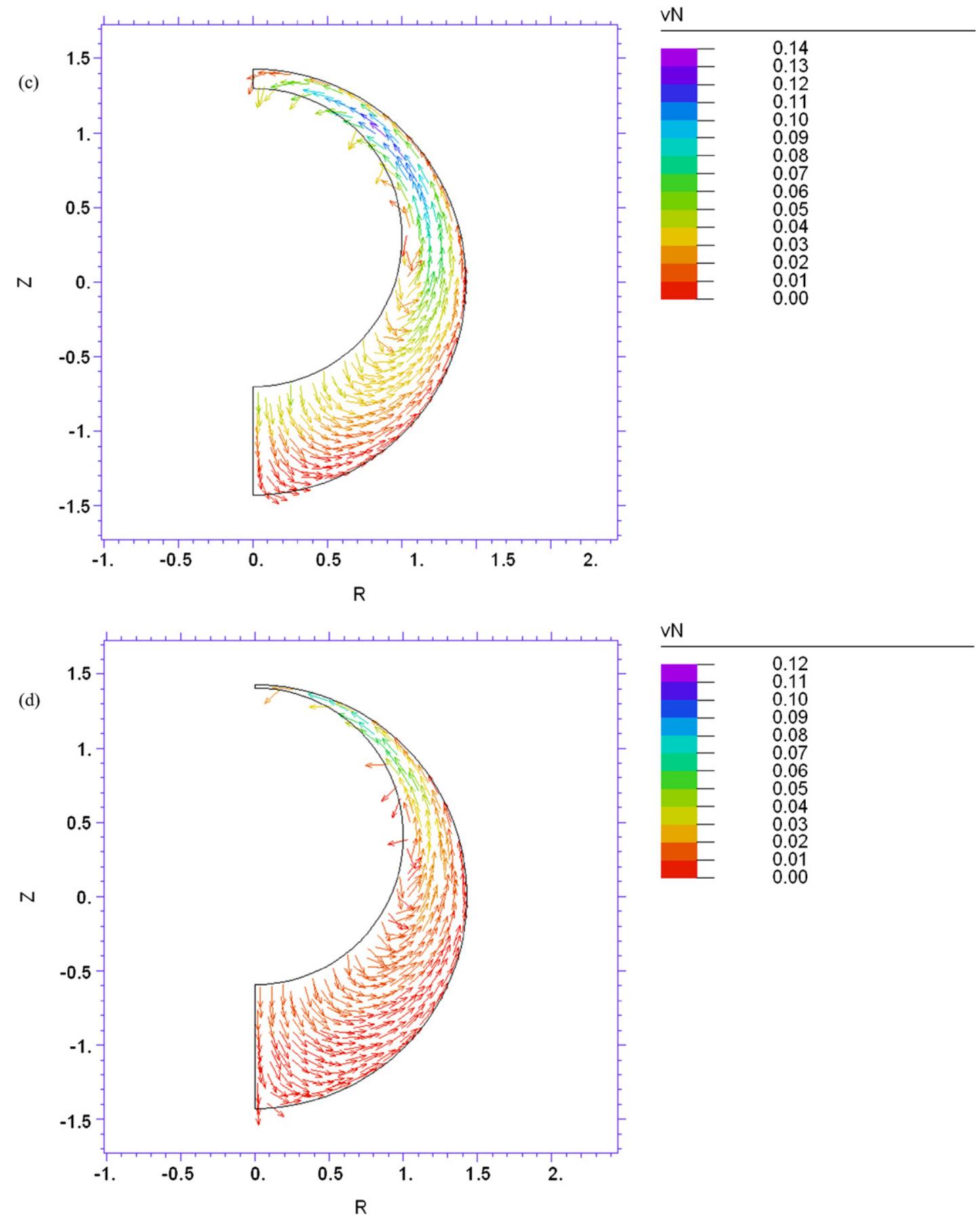

Fig. 9. (continued)

than that of the decrease of $F_{\mathrm{D}, 2(v)}^{*}$. On the other hand, the rate of decrease of $\left|F_{\mathrm{E}}^{*}\right|$ as $P$ varies is not that obvious. Fig. 11a also suggests that if $\kappa a$ is sufficiently large or it is sufficiently small, $\left|U^{*}\right|$ decreases with the increase in $P$. However, if $\kappa a$ takes a medium value, $\left|U^{*}\right|$ may have a local maximum or $U^{*}$ may changes its sign from positive to negative as $P$ increases. This is consistent with the results shown in Fig. 10a. The specific behavior of $U^{*}$ for a medium level of $\kappa a$ has not been reported in the literature for other types of geometry.

The variations of $U^{*}, F_{\mathrm{E}}^{*}$, and $F_{\mathrm{D}, 2}^{*}$ as a function of $\lambda$ at various values of $P$ when $\kappa a=1$ are presented in Fig. 12. Fig. 12a indicates that if $P$ is sufficiently large, $U^{*}$ is always negative and $\left|U^{*}\right|$ has a local maximum as $\lambda$ varies. The former is because $\left(F_{\mathrm{E}}^{*}+F_{\mathrm{D}, 2}^{*}\right)$, which is mainly dominated by $F_{\mathrm{E}}^{*}$, increases with the increase in $\lambda$, as is justified in Fig. 12b. The latter can be explained by the fact that as $\lambda$ increases, the rate of increase of $\left(F_{\mathrm{E}}^{*}+F_{\mathrm{D}, 2}^{*}\right)$ is always higher than that of $D^{*}$ if $\lambda$ is small, but the reverse is true if $\lambda$ is large. Fig. 12a also reveals that if $P$ is not too large, $U^{*}$ may change its sign from positive to negative as $\lambda$ increases and $\left|U^{*}\right|$ may have a local maximum. These behaviors are consistent with the results shown in Fig. 7 and can be explained by the same reasoning. The presence of 

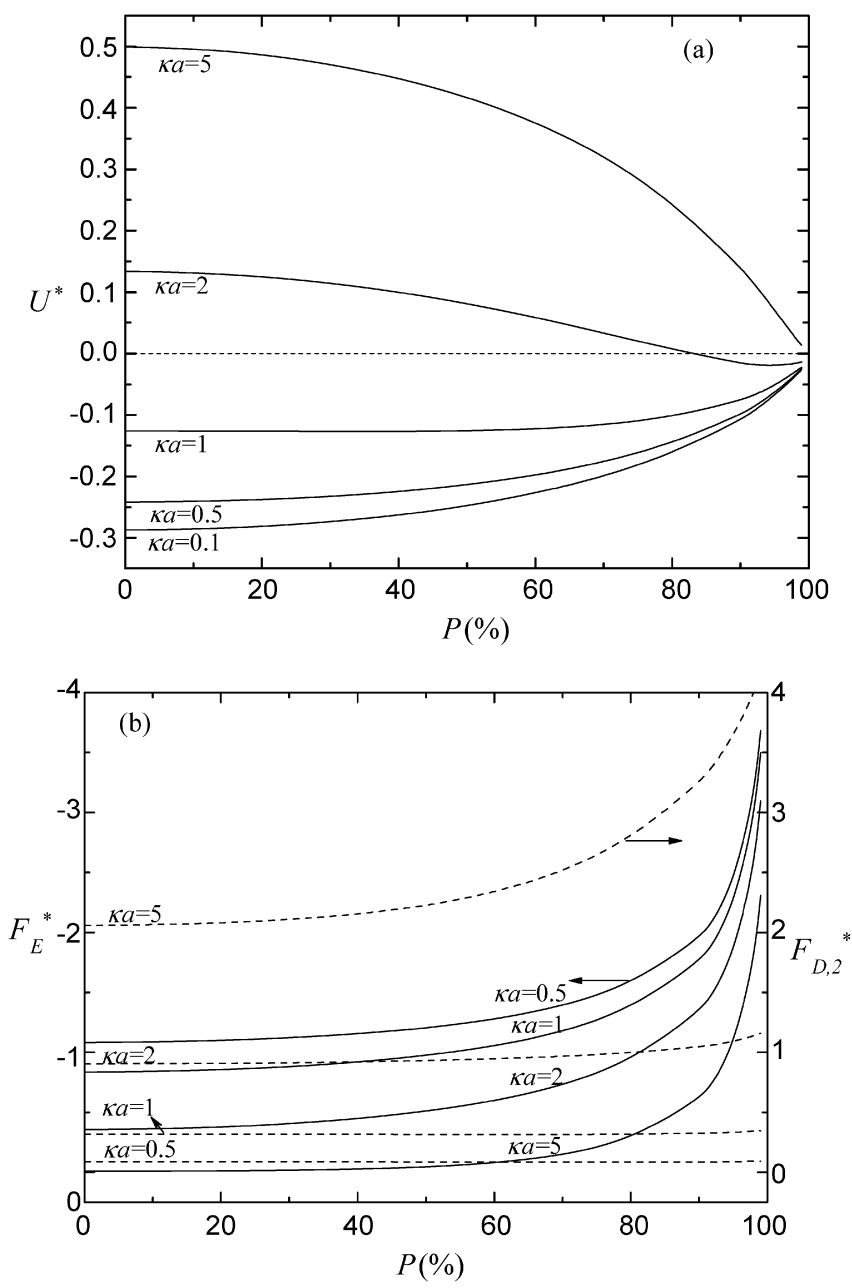

Fig. 10. Variation of (a) scaled electrophoretic mobility $U^{*}$ and (b) scaled electrostatic force $F_{\mathrm{E}}^{*}$ and scaled excess hydrodynamic force $F_{\mathrm{D}, 2}^{*}$ as functions of $P$ at various values of $\kappa a$. Key: $\zeta_{\mathrm{a}}^{*}=0, \zeta_{\mathrm{b}}^{*}=1$, and $\lambda=0.4$.

the local maximum in $\left|U^{*}\right|$ can also be explained by the fact that as $\lambda$ increases, the rate of increase of $\left(F_{\mathrm{E}}^{*}+F_{\mathrm{D}, 2}^{*}\right)$ is always higher than that of $D^{*}$ if $\lambda$ is small, but the reverse is true if $\lambda$ is large. Note that the value of $\lambda$ at which $\left|U^{*}\right|$ has a maximum increases with the decrease in $P$. This is because as $\lambda$ decreases, $-F_{\mathrm{E}}^{*}$ declines more rapidly if $P$ is larger, as is shown in Fig. 12b. Fig. 12a indicates that $U^{*} \rightarrow 0$ as $\lambda \rightarrow 1$, which is expected because if $\lambda=1$, the electroosmotic recirculation flow must vanish. Fig. 12b suggests that, regardless of the level of $P,-F_{\mathrm{E}}^{*}$ increases monotonically with the increase in $\lambda$. However, the corresponding behavior of $F_{\mathrm{D}, 2}^{*}$, which depends on the net result of the competition between $F_{\mathrm{D}, 2(v)}^{*}$ and $F_{\mathrm{D}, 2(p)}^{*}$, is more complicated. The behavior of $F_{\mathrm{D}, 2}^{*}$ can be explained by the same reasoning as that employed in the discussion of Fig. 8a. Note that as $\lambda \rightarrow 0$, Smoluchowski's result must be recovered; that is, we should have $F_{\mathrm{D}, 2}^{*} \rightarrow 1, D^{*} \rightarrow 1$, and $U^{*} \rightarrow 1$.

\section{Conclusions}

The boundary effect on electrophoresis is analyzed by considering the electrophoresis of a spherical particle at an arbi-
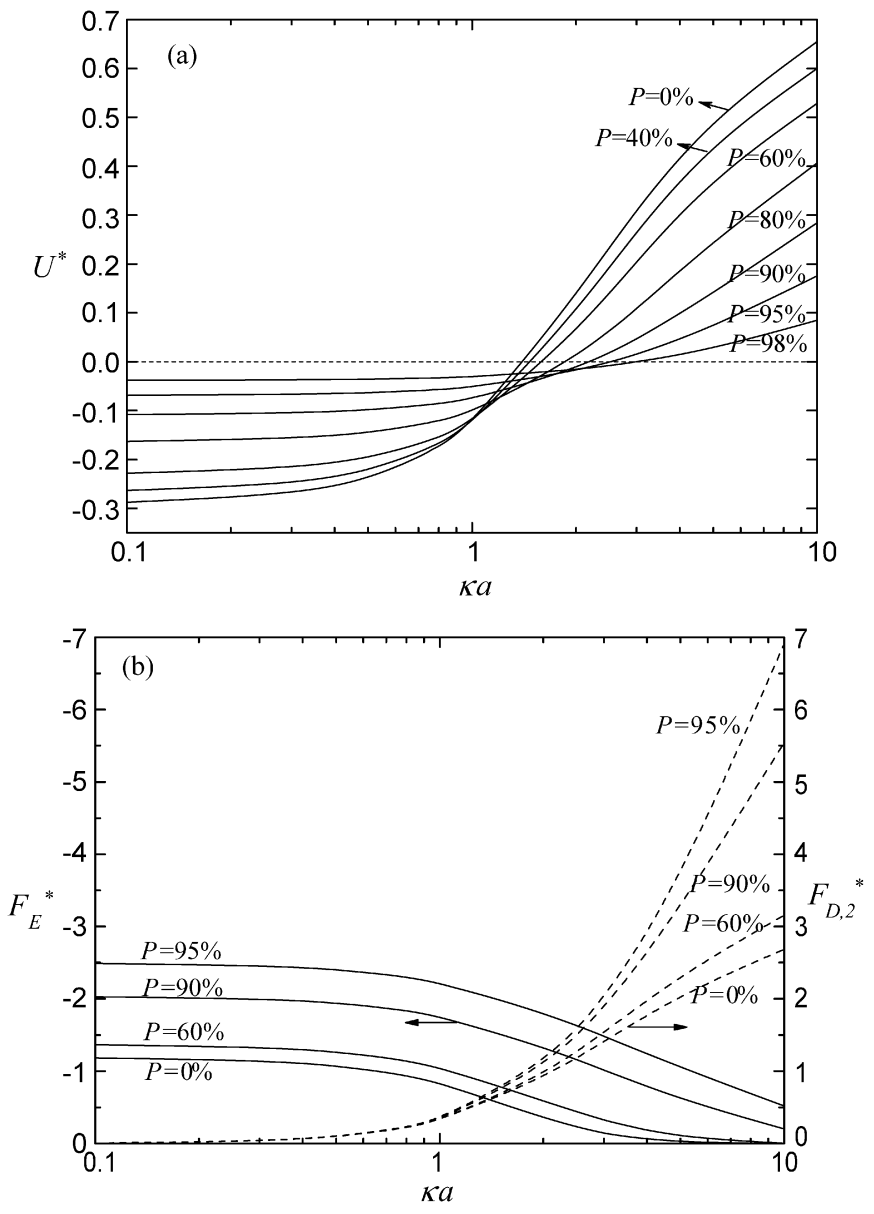

Fig. 11. Variation of (a) scaled electrophoretic mobility $U^{*}$ and (b) scaled electrostatic force $F_{\mathrm{E}}^{*}$ and scaled excess hydrodynamic force $F_{\mathrm{D}, 2}^{*}$ as functions of $\kappa a$ at various values of $P$. Key: same as in Fig. 10 .

trary position in a spherical cavity under conditions of low surface potential and weak applied electric field. For the case of a positively charged sphere in an uncharged cavity, the results obtained are consistent with those reported in the literature if a sphere is at the center of a cavity. However, if a sphere is close to the wall of a cavity, using the method in the literature for the calculation of the electrostatic force acting on a sphere will overestimate its mobility and lead to unrealistic qualitative behavior. For the case of an uncharged sphere in a positively charged cavity we conclude the following: (i) Due to the presence of an electroosmotic flow, the electrophoretic behavior of a sphere is more complicated than for the case when a positively charged sphere is placed in an uncharged cavity. (ii) The qualitative behavior of the mobility of a sphere depends upon the thickness of the double layer and how significant the boundary effect is. (iii) Even if the boundary effect is relatively unimportant, some unique electrophoretic behavior are observed that has not been reported for other types of charged boundaries.

\section{Acknowledgment}

This work is supported by the National Science Council of the Republic of China. 

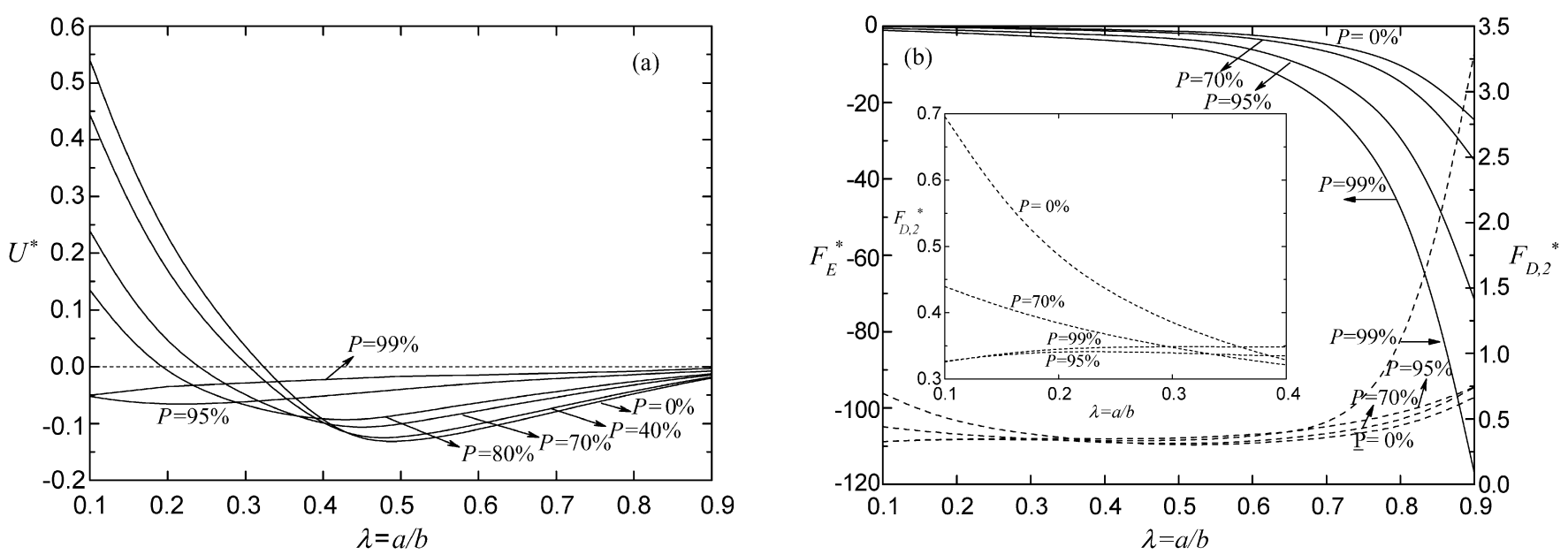

Fig. 12. Variation of (a) scaled electrophoretic mobility $U^{*}$ and (b) scaled electrostatic force $F_{\mathrm{E}}^{*}$ and scaled excess hydrodynamic force $F_{\mathrm{D}, 2}^{*}$ as functions of $\lambda$ at various values of $P$. Key: Same as in Fig. 7.

\section{References}

[1] M. Von Smoluchowski, Z. Phys. Chem. 92 (1918) 129.

[2] A.L. Zydney, J. Colloid Interface Sci. 169 (1995) 476.

[3] E. Lee, J.W. Chu, J.P. Hsu, J. Colloid Interface Sci. 196 (1997) 316.

[4] E. Lee, J.W. Chu, J.P. Hsu, J. Colloid Interface Sci. 205 (1998) 65.

[5] J.W. Chu, W.H. Lin, E. Lee, J.P. Hsu, Langmuir 17 (2001) 6289.

[6] J.P. Hsu, S.H. Hung, C.Y. Kao, Langmuir 18 (2002) 8897.

[7] J.P. Hsu, S.H. Hung, Langmuir 19 (2003) 7469.

[8] H.Y. Yu, S.H. Hung, J.P. Hsu, Colloid Polym. Sci. 283 (2004) 10.

[9] J.P. Hsu, L.H. Yeh, M.H. Ku, J. Colloid Interface Sci. 305 (2007) 324.

[10] J.P. Hsu, L.H. Yeh, J. Chin. Inst. Chem. E 37 (2006) 601.
[11] J. Ennis, J.L. Anderson, J. Colloid Interface Sci. 185 (1997) 497.

[12] A.A. Shugai, S.L. Carnie, J. Colloid Interface Sci. 213 (1999) 298.

[13] J.P. Hsu, M.H. Ku, C.Y. Kao, J. Colloid Interface Sci. 276 (2004) 248.

[14] J.P. Hsu, M.H. Ku, J. Colloid Interface Sci. 283 (2005) 592.

[15] J.P. Hsu, M.H. Ku, C.C. Kuo, Langmuir 21 (2005) 7588.

[16] D.C. Henry, Proc. R. Soc. London Ser. A 133 (1931) 106.

[17] J.P. Hsu, C.Y. Kao, J. Phys. Chem. B 106 (2002) 10605.

[18] G. Backstrom, Fluid Dynamics by Finite Element Analysis, Studentlitteratur, Sweden, 1999.

[19] R.W. O'Brien, L.R. White, J. Chem. Soc. Faraday Trans. 274 (1978) 1607.

[20] H. Ohshima, Adv. Colloid Interface Sci. 62 (1995) 189.

[21] PDE Solutions, FlexPDE, version 2.22. 